\title{
Nonlinear Projected Sparse Optimization Approach Based on Adam Algorithm for Microwave Imaging
}

\author{
Abdulla Desmal \\ Department of Electrical Engineering \\ Higher Colleges of Technology \\ Ras Al-Khaimah, UAE \\ adesmal@hct.ac.ae
}

\author{
Ali Imran Sandhu, Hakan Bagci \\ Division of Computer, Electrical, and Mathematical Science and Engineering \\ King Abdullah University of Science and Technology (KAUST) \\ Thuwal, KSA \\ \{aliimran.sandhu, hakan.bagci\}@kaust.edu.sa
}

\begin{abstract}
A microwave imaging algorithm based on contrastfield equations is developed for sparse domains. The proposed algorithm is inspired by machine learning optimization schemes. More specifically it is based on Adam approach which is a firstorder gradient optimization algorithm that has been studied intensively in optimizing artificial neural networks. To enforce sparsity constraint, the permittivity contrast at each iteration is subjected to a projection operator. The proposed algorithm has faster convergence than another state of art steepest descent approach used for microwave imaging.
\end{abstract}

Index Terms-Microwave Imaging, Inverse Scattering, Sparse Contraint, Machine Learning

\section{INTRODUCTION}

Electromagnetic (EM) imaging has been studied in the last few decades and successfully implemented in various applications ranging from medical imaging [1]-[3], crack and mine detections [4], hydrocarbon reservoir exploration [5], to through-wall imaging [6]. The forward operator of the problem that computes the scattered electric field given the permittivity profile is nonlinear. For applications with high permittivity profiles, the nonlinearity gets stronger as the multi-scattering field intensity grows to higher levels. Inverse electromagnetic imaging schemes can be classified based on their nonlinearity level. For weak scatterers with low permittivity the EM nonlinear operator is relplaced by a first order approximation like in Born approximation, Rytov approximation [7], diffraction tomography [8], and backpropagation [9]. For scatterers with high permitivitty, nonlinearity has to be accounted for fully, hence algorithms like those based on Newton [10]-[15] and nonlinear steepest descent [16], [17] are used. The full wave scattering EM schemes have been considered under two forms, the contrast-field [17], and contrast-source [10].

Besides the problem of nonlinearity, the forward operator consists of convolution smoothing integrals. Smoothining due to these integrals makes the scattered field insensitive to high frequency changes on the permittivity and renders the problem ill-conditioned [18]. To tackle the ill-conditioning, various regularization strategies have been considered. Different regularization operators make different assumptions regarding the smoothened high frequency components of the solution. Second norm regularizers remove the high frequency components from the solutions causing the reconstructed solution to have a smooth signature. However, for most applications, the reconstructed image of permittivity is not smooth, rather it contains sharp variations and/or sparse content [19]. For such problems, total variation (TV) is applied to promote profiles with sharp variations or piece-wise discontinuity signature [20]. On the other hand, for sparse images, images with few non-zero pixels, the first and zero norms have been adapted to promote sparseness of the solution [13], [17], [21].

In this work, a full wave inverse scattering scheme is proposed based on accelerated version of nonlinear steepest descent algoritm known as ADAM [22] which has been intensively studied to find the hyperparameters of artificial neural networks. The scattering equations are formulated in the contrast-field form. To enforce the sparsity constraint, the solution in each iteration is projected into a first-norm constraint subset.

The rest of the paper is organized as follow. Section-II-A describes the forward EM scattering problem that express the scatteres field in term of the material properties. Section-II-B discusses the optimization problem assuming sparse investigation domain. Section-II-C introduces the projected sparse ADAM algorithm. Section-III provides numerical examples to compare proposed scheme to the well-known nonlinear Landweber algorithm.

\section{FORMULATION}

\section{A. EM forward model}

Fig. 1 shows the problem configuration. An investigation domain $D^{i n v}$ that contains the object(s) under test is discretized into $N$-cells. $N^{\mathrm{T}}$ number of line source (transmitter) antennas polarized along z-axis illuminate $D^{i n v}$ from multiple angles around $D^{i n v}$. Due to the object(s) inside $D^{i n v}$, the incident fields are scattered and measured at $N^{\mathrm{R}}$ number of receivers locations (represented as domain $D^{\text {meas }}$ ). The measured scattered fields for a single source excitation are expressed as:

$$
\overline{\mathbf{E}}_{j}^{s c a}=f_{j}(\overline{\mathbf{t}})=\overline{\mathbf{H}}(\overline{\mathbf{I}}-\overline{\mathbf{F}} D\{\overline{\mathbf{t}}\})^{-1} \overline{\mathbf{E}}_{j}^{i n c}
$$

where $\overline{\mathbf{E}}_{j}^{s c a}$ is $N^{\mathrm{R}} \times 1$ vector, that stores the scattered field for all receivers due to transmitter $j, j \in\left\{1,2, \ldots, N^{\mathrm{T}}\right\}$. $\overline{\mathbf{H}}$ 


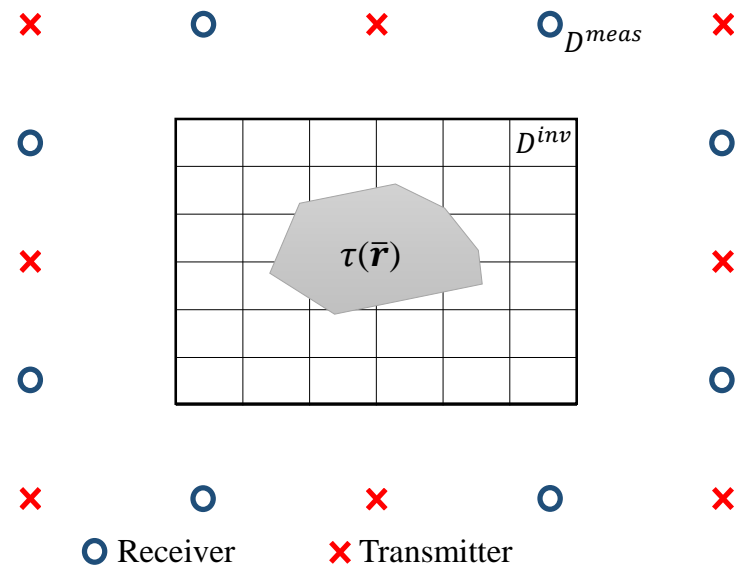

Fig. 1. Problem configuration.

is $N^{\mathrm{R}} \times N$ observation matrix, and $\overline{\mathbf{F}}$ is $N \times N$ data matrix. The elements of $\overline{\mathbf{H}}$ and $\overline{\mathbf{F}}$ are expressed as

$$
\begin{array}{ll}
\overline{\mathbf{H}}_{(m, n)}=k_{0}^{2} \int_{\mathbf{r}^{\prime} \in S^{n}} G_{2 D}\left(\mathbf{r}_{m}, \mathbf{r}^{\prime}\right) d S^{\prime}, & \mathbf{r}_{m} \in D^{\text {meas }} \\
\overline{\mathbf{F}}_{(m, n)}=k_{0}^{2} \int_{\mathbf{r}^{\prime} \in S^{n}} G_{2 D}\left(\mathbf{r}_{m}, \mathbf{r}^{\prime}\right) d S^{\prime}, & \mathbf{r}_{m} \in D^{\text {inv }}
\end{array}
$$

where $G_{2 D}\left(\mathbf{r}, \mathbf{r}^{\prime}\right)=H_{0}^{(2)}\left(k_{0}\left|\mathbf{r}-\mathbf{r}^{\prime}\right|\right) / 4 j$ is the 2D scalar Green function, $k_{0}=\omega \sqrt{\mu_{0} \epsilon_{0}}$ is the background wavenumber, $\omega$ is the angular frequency, $\mu_{0}$ is the background permeability, and $\epsilon_{0}$ is the background permittivity. In (1), $\overline{\mathbf{t}}$ is an $N \times 1$ vector stores entries of the contrast values of the discretized cells in $D^{i n v}$. The operator $D\{$.$\} produces a diagonal matrix$ with entries equal to the input vector. Finally, $\overline{\mathbf{E}}_{j}^{i n c}$ is $N \times 1$ vector stores entries of the incident field of the $j^{\text {th }}$ transmitter computed at the centers of the discretized cells on $D^{i n v}$. To account for all scattered fields generated by all the transmitters, Eq. (1) is cascaded for all $j$

$$
\begin{gathered}
\overline{\mathbf{E}}^{s c a}=\left[\overline{\mathbf{E}}_{1}^{s c a}, \overline{\mathbf{E}}_{2}^{s c a}, \ldots . ., \overline{\mathbf{E}}_{N^{\mathrm{T}}}^{s c a}\right] \\
f(\overline{\mathbf{t}})=\left[f_{1}(\overline{\mathbf{t}}), f_{2}(\overline{\mathbf{t}}), \ldots . ., f_{N^{\mathrm{T}}}(\overline{\mathbf{t}})\right] .
\end{gathered}
$$

\section{B. Sparse Optimization}

In this work the sparse optimization problem is constructed as [23]

$$
\arg \min _{\overline{\mathbf{t}}}\left\|f(\overline{\mathbf{t}})-\overline{\mathbf{E}}^{\text {meas }}\right\|_{2}^{2} \quad \text { s.t. }|\overline{\mathbf{t}}|_{1} \leq l_{1}
$$

where $\overline{\mathbf{E}}^{\text {meas }}=\overline{\mathbf{E}}^{s c a}+\bar{\eta}$ is the scattered field measured the receiver locations and is assumed to be equal to the computed scattered field $\overline{\mathbf{E}}^{s c a}$ plus additive white Gaussian noise $\bar{\eta}$. The optimization problem in (3) minimizes the data misfit or data discrepancy subjected to a first norm constraint of the solution bounded by $l_{1}$ ball.

\section{Projected Adam}

The nonlinear least square minimization in (3) is carried out using an enhanced nonlinear steepest descent algorithm known as Adam which has been explored intensively in training artificial neural networks [22]. Further, the first-norm sparseness constraint is enforced via a thresholding projection operator [17]. The proposed projected Adam algorithm to solve the optimization problem (3) reads as follow:

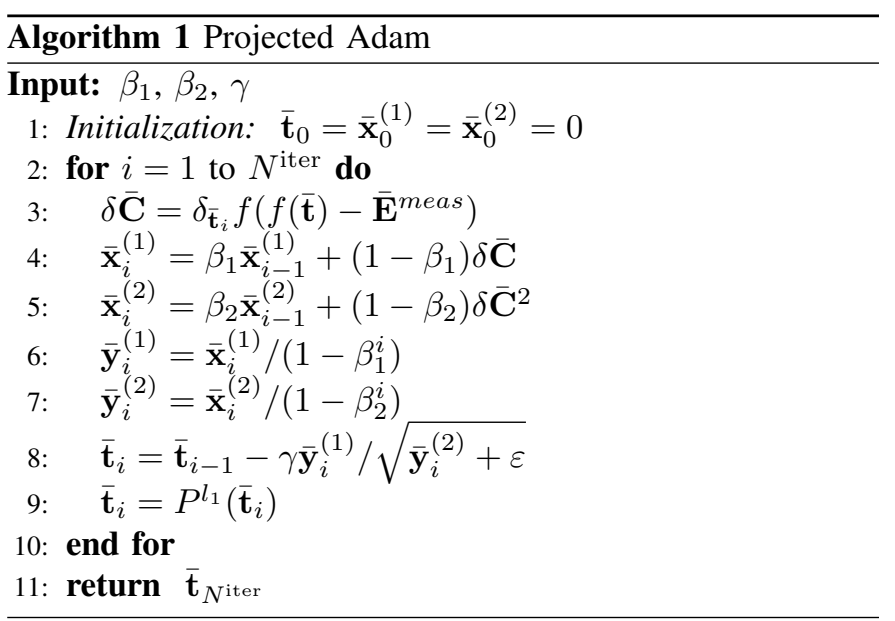

The parameters $\beta_{1}$ and $\beta_{2}$ are selected to determine the first and second momentum effect on the iterative solution which is thought as an object while the gradient is thought as its velocity vector. The gradient at each iteration is computed at Step 3. Steps 4-5 compute the first and second momentum effects while Step 8 computes the iterative solution based on the first and second momentum effects. Step 9 is the projection of the solution $\overline{\mathbf{t}}_{i}$ into first norm ball with $l_{1}$ radius. The algorithm of the projection operator is detailed next:

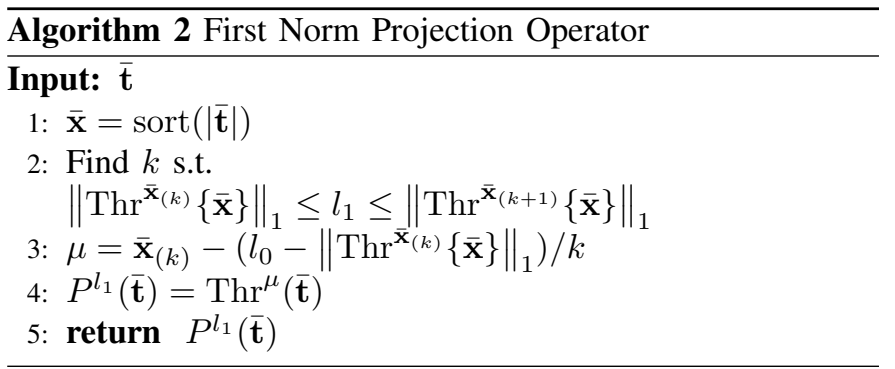

Step 1 sorts the input vector $\overline{\mathrm{t}}$ in a descent manner based on its absolute values. At $\operatorname{Step~2,} \operatorname{Thr}^{\delta}(\overline{\mathbf{x}})$ is a soft thresholding operator with thresholding level $\delta$ and is computed using [23]:

$$
\left\{\operatorname{Thr}^{\delta}(\overline{\mathbf{x}})\right\}_{(n)}=\{\overline{\mathbf{x}}\}_{(n)} \frac{\max \left\{\left|\{\overline{\mathbf{x}}\}_{(n)}-\delta\right|, 0\right\}}{\max \left\{\left|\{\overline{\mathbf{x}}\}_{(n)}-\delta\right|, 0\right\}+\delta}
$$

Step 2 searches for index $k$ such that the first norm ball $l_{1}$ will lie within the first norm thresholding operator with $\overline{\mathbf{x}}_{(k+1)}$ and $\overline{\mathbf{x}}_{(k)}$ as their thresholding levels. Step 3 computes the required level of thresholding $\mu$ that achieves $l_{1}$ projection. Finally. Step 4 executes this thresholding.

\section{NUMERICAL RESUlts}

In this section the projected Adam algorithm is compared to an enhanced version of thresholded nonlinear landweber (NLW) iterations using a numerical expermint [16]. The enhanced NLW version uses the projection operator described in this work to promote sparseness in the solution rather than 


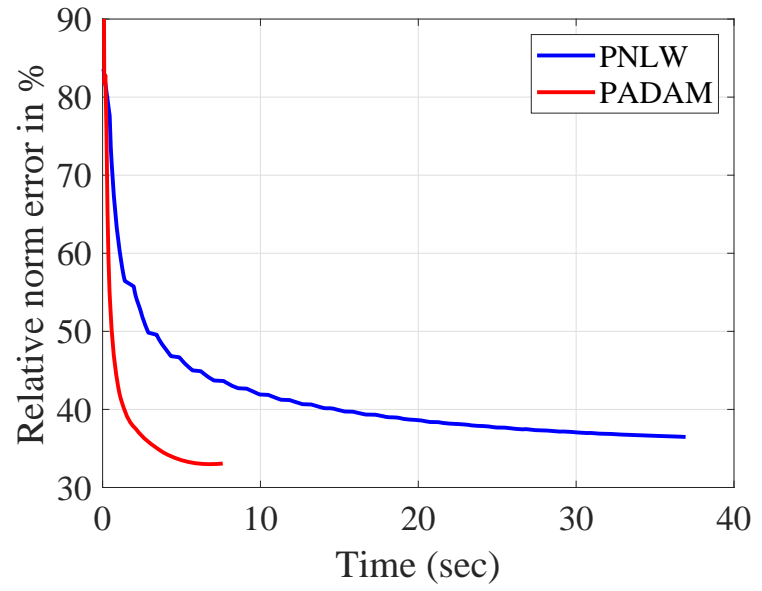

(a)

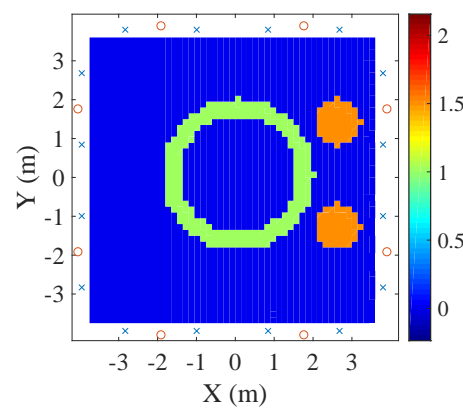

(b)

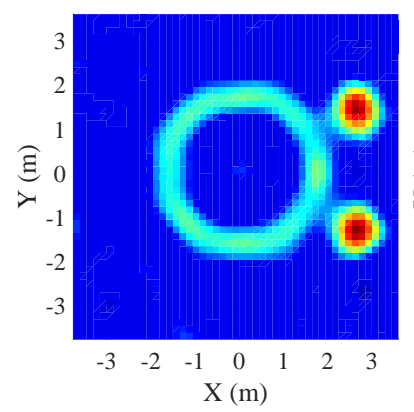

(d)

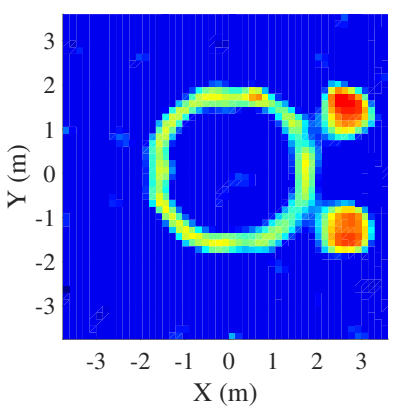

(c)

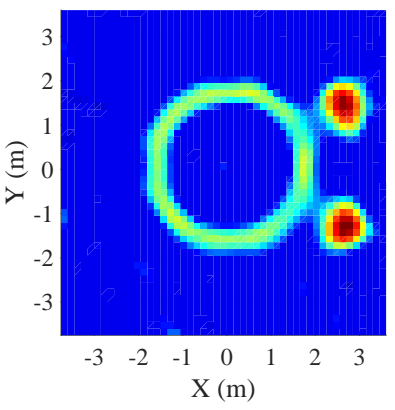

(e)
Fig. 2. (a) Relative reconstruction error for PADAM and PNLW. (b) Actual permitivity profile for Austria along with transmitter-receiver locations (c) PADAM reconstruction profile at 7.54 seconds with $33 \%$ relative norm error. (d) PNLW reconstruction profile at 7.5 seconds with $43.7 \%$ relative norm error. (e) PNLW reconstruction profile on convergance at 36.9 seconds.

simply imposing soft-thresholding. The use of projected NLW is indeed show that the improvement is due to the nature of Adam algorithm rather than the projection operation.

Figure 2 shows the reconstruction results for the Austria profile. Fig. 2(a) shows the relative norm error for the projected Adam (PADAM) and projected nonlinear Landweber (PNLW). Fig. 2(b) shows the actual profile of Austria that has been used to generate measurments with $25 \mathrm{~dB}$ noise. Fig. 2(c) shows the PADAM reconstruction profile at $7.54 \mathrm{sec}$ while fig. 2(d) shows the reconstruction profile around the same time obtained using PNLW approach. Finally, fig. 2(e) shows
PNLW at convergence which is obtained after 36.9 sec.

\section{REFERENCES}

[1] X. Song, M. Li, F. Yang, S. Xu, and A. Abubakar, "Study on joint inversion algorithm of acoustic and electromagnetic data in biomedical imaging," IEEE Journal on Multiscale and Multiphysics Computational Techniques, vol. 4, pp. 2-11, 2019.

[2] N. Abdollahi, D. Kurrant, P. Mojabi, M. Omer, E. Fear, and J. LoVetri, "Incorporation of ultrasonic prior information for improving quantitative microwave imaging of breast," IEEE Journal on Multiscale and Multiphysics Computational Techniques, vol. 4, pp. 98-110, 2019.

[3] G. Chen, P. Shah, J. Stang, and M. Moghaddam, "Learning-assisted multi-modality dielectric imaging," IEEE Transactions on Antennas and Propagation, 2019.

[4] S. Caorsi, A. Massa, M. Pastorino, and F. Righini, "Crack detection in lossy two-dimensional structures by means of a microwave imaging approach," International Journal of Applied Electromagnetics and Mechanics, vol. 11, no. 4, pp. 233-244, 2000.

[5] A. Desmal and H. Bağc1, "Nonlinear microwave imaging using levenberg-marquardt method with iterative shrinkage thresholding," in 2014 USNC-URSI Radio Science Meeting (Joint with AP-S Symposium). IEEE, 2014, pp. 252-252.

[6] F. Soldovieri, R. Solimene, and G. Prisco, "A multiarray tomographic approach for through-wall imaging," IEEE Transactions on Geoscience and Remote Sensing, vol. 46, no. 4, pp. 1192-1199, 2008.

[7] B. Chen and J. J. Stamnes, "Validity of diffraction tomography based on the first born and the first rytov approximations," Applied optics, vol. 37, no. 14, pp. 2996-3006, 1998.

[8] W. Tabbara, B. Duchêne, C. Pichot, D. Lesselier, L. Chommeloux, and N. Joachimowicz, "Diffraction tomography: contribution to the analysis of some applications in microwaves and ultrasonics," Inverse Problems, vol. 4, no. 2, p. 305, 1988.

[9] P. M. van den Berg, A. Van Broekhoven, and A. Abubakar, "Extended contrast source inversion," Inverse problems, vol. 15 , no. 5, p. 1325 , 1999.

[10] G. Bozza and M. Pastorino, "An inexact Newton-based approach to microwave imaging within the contrast source formulation," IEEE Trans. Antennas Propag., vol. 57, no. 4, pp. 1122-1132, 2009.

[11] C. Estatico, M. Pastorino, and A. Randazzo, "An inexact-newton method for short-range microwave imaging within the second-order born approximation," IEEE Trans. Geosci. Remote Sens., vol. 43, no. 11, pp. 2593-2605, 2005.

[12] G. Bozza, C. Estatico, M. Pastorino, and A. Randazzo, "An inexact newton method for microwave reconstruction of strong scatterers," IEEE Antennas Wireless Propag. Lett., vol. 5, no. 1, pp. 61-64, 2006.

[13] A. Desmal and H. Bagci, "A preconditioned inexact Newton method for nonlinear sparse electromagnetic imaging," IEEE Trans. Geosci. Remote Sens. Lett., vol. 12, no. 3, pp. 532-536, 2015.

[14] A. Desmal and H. Bagci, "Sparse electromagnetic reconstruction via iterative shrinkage thresholding algorithms," in Proc. Appl. Computational Electromagn. Soc. Symp., Jacksonville, FL, March 2014.

[15] A. Desmal and H. Bağc1, "Sparse electromagnetic imaging using nonlinear iterative shrinkage thresholding," in 2015 9th European Conference on Antennas and Propagation (EuCAP). IEEE, 2015, pp. 1-4.

[16] A. Desmal and H. Bagci, "Sparse electromagnetic imaging using nonlinear Landweber iterations," Prog. Electromagn. Res., vol. 152, pp. 77-93, 2015.

[17] A. Desmal and H. Bağcı, "Sparse nonlinear electromagnetic imaging accelerated with projected steepest descent algorithm," IEEE Trans. Geosci. Remote Sens., vol. 55, no. 7, pp. 3810-3822, 2017.

[18] M. Pastorino, Microwave Imaging. Wiley, 2010.

[19] G. Oliveri, N. Anselmi, and A. Massa, "Compressive sensing imaging of non-sparse $2 \mathrm{~d}$ scatterers by a total-variation approach within the born approximation," IEEE Trans. Antennas Propag., vol. 62, no. 10, pp. 5157-5170, 2014

[20] A. Abubaker and P. M. Van Den Berg, "Total variation as a multiplicative constraint for solving inverse problems," IEEE Transactions on Image Processing, vol. 10, no. 9, pp. 1384-1392, 2001.

[21] A. Desmal and H. Bagci, "Shrinkage-thresholding enhanced Born iterative method for solving 2D inverse electromagnetic scattering problem," IEEE Trans. Antennas Propag., vol. 62, no. 7, pp. 3878-3884, 2014.

[22] D. P. Kingma and J. Ba, "Adam: A method for stochastic optimization," arXiv preprint arXiv:1412.6980, 2014. 
[23] I. Daubechies, M. Fornasier, and I. Loris, "Accelerated projected gradient method for linear inverse problems with sparsity constraints," J. Fourier Anal. Appl., vol. 14, no. 5-6, pp. 764-792, 2008. 